\title{
Glaucocalyxin A alleviates lipopolysaccharide-induced inflammation and apoptosis in pulmonary microvascular endothelial cells and permeability injury by inhibiting STAT3 signaling
}

\author{
JIANWEI CAO, MEILING LIU, SHUFANG FENG，YINGYING LI and KAIJUN ZHENG \\ Pediatrics Department, Zhongshan People's Hospital, Zhongshan, Guangdong 528403, P.R. China
}

Received May 27, 2021; Accepted October 29, 2021

DOI: $10.3892 /$ etm.2022.11242

\begin{abstract}
Glaucocalyxin A (GLA), an ent-kauranoid diterpene derived from Rabdosia japonica var. glaucocalyx, possesses antibacterial, anti-oxidative and anti-neuroinflammatory properties. The present study aimed to investigate the potential mechanisms underlying GLA in the pathogenesis of pneumonia. Human pulmonary microvascular endothelial cells (hPMVECs) treated with lipopolysaccharide (LPS) were treated with GLA, followed by the detection of cell viability, inflammation, apoptosis and cell permeability. Furthermore, the protein expression levels of apoptosis- and permeability-associated proteins were determined using western blot analysis. Following treatment with a signal transducer and activator of transcription 3 (STAT3) activator, the protein expression levels of STAT3 and endoplasmic reticulum stress-associated proteins were determined, to confirm whether STAT3 signaling was mediated by GLA. Lastly, the mRNA expression level of inflammatory cytokines, apoptosis and permeability injury were also determined following treatment with a STAT3 activator. The results revealed that GLA ameliorated inflammation, apoptosis and permeability injury in LPS-induced hPMVECs. Following treatment with a STAT3 activator, the therapeutic effects of GLA on LPS-induced hPMVECs were abrogated. In conclusion, GLA alleviated LPS-induced inflammation, apoptosis and permeability injury in hPMVECs by inhibiting STAT3 signaling, which highlighted the potential therapeutic value of GLA in the treatment of pneumonia.
\end{abstract}

\section{Introduction}

Pneumonia is a respiratory disease characterized by lung inflammation that is mainly caused by pathogens (1). The

Correspondence to: Mr. Kaijun Zheng, Pediatrics Department, Zhongshan People's Hospital, 2 East-Sun-Wen Road, Zhongshan, Guangdong 528403, P.R. China

E-mail: cjwinzs@163.com

Key words: glaucocalyxin A, STAT3, inflammation, apoptosis main features of pneumonia include fever, cough, dyspnea, somnolence and chest pain, and is a common disease in children, accounting for 24.5-65.2\% of pediatric inpatients (2-4). Lipopolysaccharide (LPS), the major bioactive component of the cell wall, serves a pivotal role in inducing an inflammatory response in patients with pneumonia (5). Despite substantial advances in the therapeutic approaches to pneumonia, further investigation is required (6). Thus, current research should focus on the mechanisms underlying the inflammatory response and aim to identify novel treatment options for pneumonia. Emerging evidence has indicated that inflammation-related proteins, including TNF- $\alpha$, IL-1 $\beta$ and IL- 6 , as well as apoptosis-related proteins Bcl-2 and Bax, were associated with LPS-induced acute pneumonia (7-10).

Glaucocalyxin A (GLA), an ent-kauranoid diterpene derived from Rabdosia japonica var. glaucocalyx, possesses a number of antibacterial, anti-oxidative and anti-neuroinflammatory properties $(11,12)$. The results of a previous study revealed the therapeutic potential of GLA in the treatment of LPS-mediated septic shock and inflammation, in which GLA suppressed the activation of the NACHT, LRR and PYD domains-containing protein 3 inflammasome (13). Furthermore, GLA attenuated myocardial ischemia/reperfusion injury by alleviating microvascular thrombosis in a mouse model (14). HY1702, a novel small molecule diterpene acquired from the modification of GLA, reduced the injury of acute respiratory distress syndrome in mice induced by LPS (15). GLA also alleviated pulmonary fibrosis in mice, which improved the survival rates of mice with this disease (16). The results of previous studies demonstrated that GLA possessed anti-inflammatory and anti-oxidative stress effects $(17,18)$. Furthermore, GLA inhibited the activation of signal transducer and activator of transcription 3 (STAT3) in osteosarcoma cells, and STAT3 inhibition alleviated LPS-induced acute lung injury (19-21). In addition, a previous study demonstrated that STAT3 binds to the promoter site of activating transcription factor 6 to increase its expression, thereby inducing endoplasmic reticulum stress (ERS) (22). Therefore, the present study aimed to investigate the role of GLA in ERS inhibition regulated by ATF6 and the effects on STAT3 activity. In addition, the role of GLA in LPS-induced inflammation and apoptosis in human pulmonary 
microvascular endothelial cells (hPMVECs) and endothelial cell permeability injury was also analyzed.

\section{Materials and methods}

Cell lines and treatment. GLA was purchased from MedChemExpress. Immortalized hPMVECs were obtained from The Cell Bank of Type Culture Collection of The Chinese Academy of Sciences (https://www.biomart.cn/infosupply/33021544.htm?from=search_1) and cultured in DMEM, supplemented with $10 \% \mathrm{FBS}$ and $1 \%$ penicillin/streptomycin (all, Invitrogen; Thermo Fisher Scientific, Inc.), at $37^{\circ} \mathrm{C}$ in an incubator with $5 \% \mathrm{CO}_{2}$. LPS (Sigma-Aldrich; Merk $\mathrm{KGaA}$; resuspended in DMEM) was added to the cells for cell model establishment, at a concentration of $100 \mathrm{ng} / \mathrm{ml}$ for $24 \mathrm{~h}$. In subsequent experiments, 1.25, 2.5, 5 and $10 \mu \mathrm{M}$ GLA (MedChemExpress; resuspended in $100 \mathrm{mg} / \mathrm{m}$ DMSO) and $0.5 \mu \mathrm{M}$ colivelin (MedChemExpress; resuspended in DMEM), which is an activator of STAT3, were used for the treatment of the cells $(18,23,24)$.

Cell Counting Kit (CCK)-8 assay. Briefly, hPMVECs were seeded in 96 -well plates, at a density of $3 \times 10^{5}$ cells/well. After the cells were treated with GLA, $10 \mu \mathrm{l} \mathrm{CCK-8} \mathrm{reagent}$ (Dojindo Molecular Technologies, Inc.) was added to the cells and incubated for $4 \mathrm{~h}$, according to the manufacturer's protocol. Cell viability was determined by measuring the optical density at $450 \mathrm{~nm}$ using a microplate reader (BioTek Instruments, Inc.).

Reverse transcription-quantitative (RT-q) PCR analysis. Following LPS and GLA treatment, total RNA was extracted from the hPMVECs using TRIzol ${ }^{\circledR}$ (Invitrogen; Thermo Fisher Scientific, Inc.). Single-stranded cDNA was subsequently synthesized from total RNA using the PrimeScript $^{\mathrm{TM}}$ RT Reagent kit, with gDNA Eraser (cat. no. RR047A; Takara Biotechnology Co., Ltd.) according to the manufacturer's protocol. SYBR Green qPCR Master Mix (MedChemExpress) was used for qPCR. The following thermocycling conditions were used for qPCR: Initial denaturation for $2 \mathrm{~min}$ at $95^{\circ} \mathrm{C}$; followed by 40 cycles at $94^{\circ} \mathrm{C}$ for $30 \mathrm{sec}, 60^{\circ} \mathrm{C}$ for $30 \mathrm{sec}$ and $72^{\circ} \mathrm{C}$ for $2 \mathrm{~min}$; and a final extension at $72^{\circ} \mathrm{C}$ for $5 \mathrm{~min}$. mRNA expression levels were determined using the $2^{-\triangle \Delta C q}$ method (25) and GAPDH was used as the internal control for quantitative normalization of mRNA. The following primers were used: TNF- $\alpha$ forward, 5'-CCT CTCTCTAATCAGCCCTCTG-3' and reverse, 5'-GAGGAC CTGGGAGTAGATGAG-3'; IL-1 $\beta$ forward, 5'-ATGATG GCTTATTACAGTGGCAA-3' and reverse, 5'-GTCGGA GATTCGTAGCTGGA-3', IL-6, forward, 5'-ACTCACCTC TTCAGAACGAATTG-3' and reverse, 5'-CCATCTTTG GAAGGTTCAGGTTG-3'; GAPDH forward, 5'-GGAGCG AGATCCCTCCAAAAT-3' and reverse, 5'-GGCTGTTGT CATACTTCTCATGG-3'.

TUNEL assay. A TUNEL assay was used to detect the apoptosis of cultured hPMVECs. After the cells were fixed with $4 \%$ paraformaldehyde at room temperature for $30 \mathrm{~min}$, a TUNEL assay kit (Roche Diagnostics $\mathrm{GmbH}$ ) was used to detect the apoptotic cells according to the manufacturer's protocol.
Apoptotic cells of each section were stained using $50 \mu \mathrm{l}$ TUNEL at $37^{\circ} \mathrm{C}$ for $60 \mathrm{~min}$. Following nuclear staining with DAPI $(0.1 \mu \mathrm{g} / \mathrm{ml})$ at room temperature for $5 \mathrm{~min}$ in the dark, Antifade mounting medium was used to mount the sections (Beyotime Institute of Biotechnology). Fluorescent images taken at four random fields of view were captured using an inverted microscope and subsequently analyzed using ImageJ (v1.38; National Institutes of Health).

Western blot analysis. Total protein was extracted from the hPMVECs using RIPA Lysis Buffer (Beyotime Institute of Biotechnology) according to the manufacturer's protocol. Total protein was quantified using a BCA kit (Beyotime Institute of Biotechnology) and the proteins (30 $\mu \mathrm{g}$ per lane) were separated using $12 \%$ SDS-PAGE. The separated proteins were subsequently transferred onto PVDF membranes and blocked with $5 \%(\mathrm{w} / \mathrm{v})$ skimmed milk for $2 \mathrm{~h}$ at room temperature. The membranes were incubated overnight at $4^{\circ} \mathrm{C}$ with the following primary antibodies at 1:1,000 dilution: Anti-Bcl-2 (cat. no. ab32124), anti-Bax (cat. no. ab32503), anti-vascular endothelial (VE)-cadherin (cat. no. ab232880), anti-zona occludens protein-1 (ZO-1; cat. no. ab216880), anti-claudin 5 (cat. no. ab131259), anti-phosphorylated (p)-STAT3 (cat. no. ab76315), anti-STAT3 (cat. no. ab68153), anti-ATF6 (a cat. no. b122897), anti-C/EBP-homologous protein (CHOP; cat. no. ab11419), anti-glucose regulated protein 78 (GRP78; cat. no. ab21685) and anti-GAPDH (cat. no. ab9485) (all from Abcam). The membranes were subsequently incubated with horseradish peroxidase-conjugated secondary antibodies (cat. no. sc-2357; 1:10,000; Santa Cruz Biotechnology, Inc.) for $1 \mathrm{~h}$ at room temperature. GAPDH was used as an internal control. Protein bands were visualized using ECL (Thermo Fisher Scientific, Inc.).

FITC-dextran assay. The FITC-dextran assay was performed as previously described (26). Briefly, monolayers were cultured using the treated hPMVECs with LPS combined with GLA or GLA and colivelin at $2 \times 10^{5}$ cells $/ \mathrm{cm}^{2}$ at $37^{\circ} \mathrm{C}$. Subsequently, $500 \mu \mathrm{g} / \mathrm{ml}$ FITC-dextran was added to the apical compartment of the transwell chamber (Corning, Inc.), and $200 \mu 1$ samples from serum-free DMEM (lower chamber) were collected $2 \mathrm{~h}$ later at $37^{\circ} \mathrm{C}$, following the addition of FITC-dextran. The absorbance was measured using a microplate reader with excitation at $485 \mathrm{~nm}$ and emission at $535 \mathrm{~nm}$.

Statistical analysis. The data are presented as the mean \pm SD. The comparisons between more than two groups were assessed using one-way ANOVA followed by Tukey's post hoc test, using GraphPad Prism 5 (GraphPad Software Inc.). Each experiment was repeated at least three times. $\mathrm{P}<0.05$ was considered to indicate a statistically significant difference.

\section{Results}

GLA ameliorates LPS-induced hPMVEC injury. The dysfunction of endothelial cells has been associated with the pathophysiological consequences of acute lung injury (27). To investigate the role of GLA in acute pneumonia, hPMVECs were treated with different concentrations of GLA to observe the corresponding cytotoxicity. As exhibited in Fig. 1A, low, 

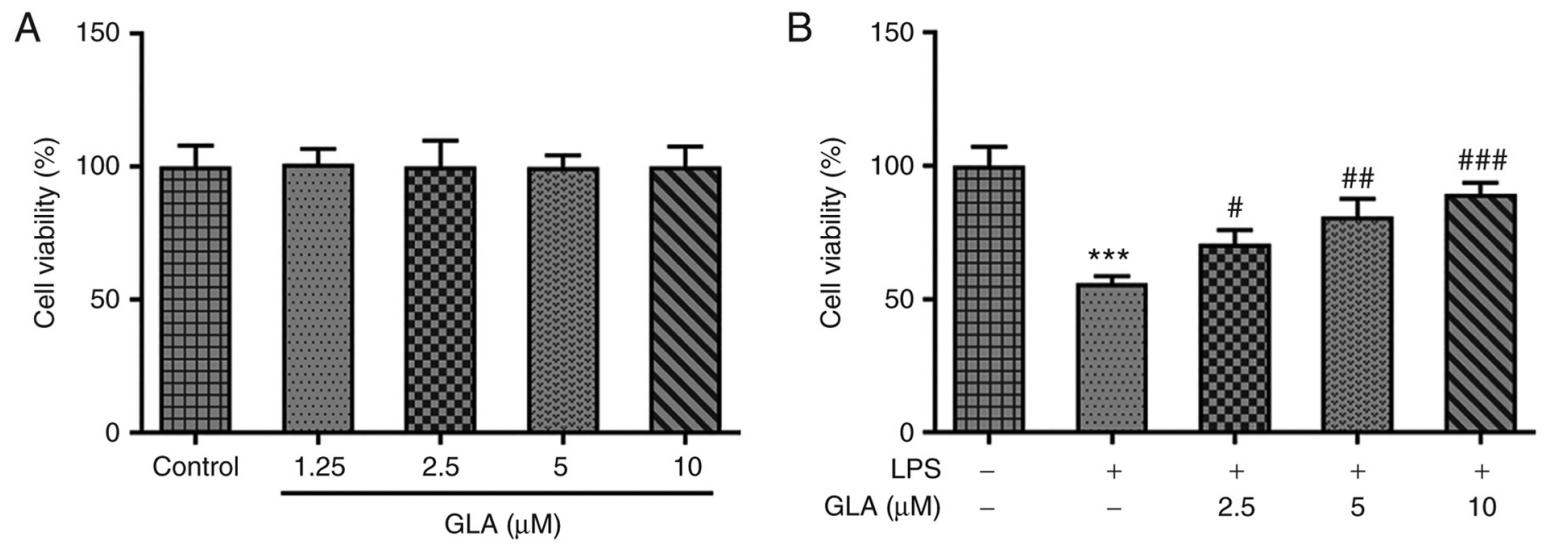

Figure 1. GLA ameliorates LPS-induced hPMVEC injury. Cell viability of (A) hPMVECs treated with different concentrations of GLA was determined by performing a Cell Counting Kit-8 assay and (B) hPMVECs treated with GLA after LPS induction. ${ }^{* * *} \mathrm{P}<0.001$ vs. LPS $(-)+$ GLA $(\mu \mathrm{M})(-) ;{ }^{\# P} \mathrm{P}<0.05$, ${ }^{\# \#} \mathrm{P}<0.01$ and ${ }^{\# \# \#} \mathrm{P}<0.001$ vs. LPS $(+)+$ GLA $(\mu \mathrm{M})(-)$. hPMVECs, human pulmonary microvascular endothelial cells; GLA, glaucocalyxin A; LPS, lipopolysaccharide.
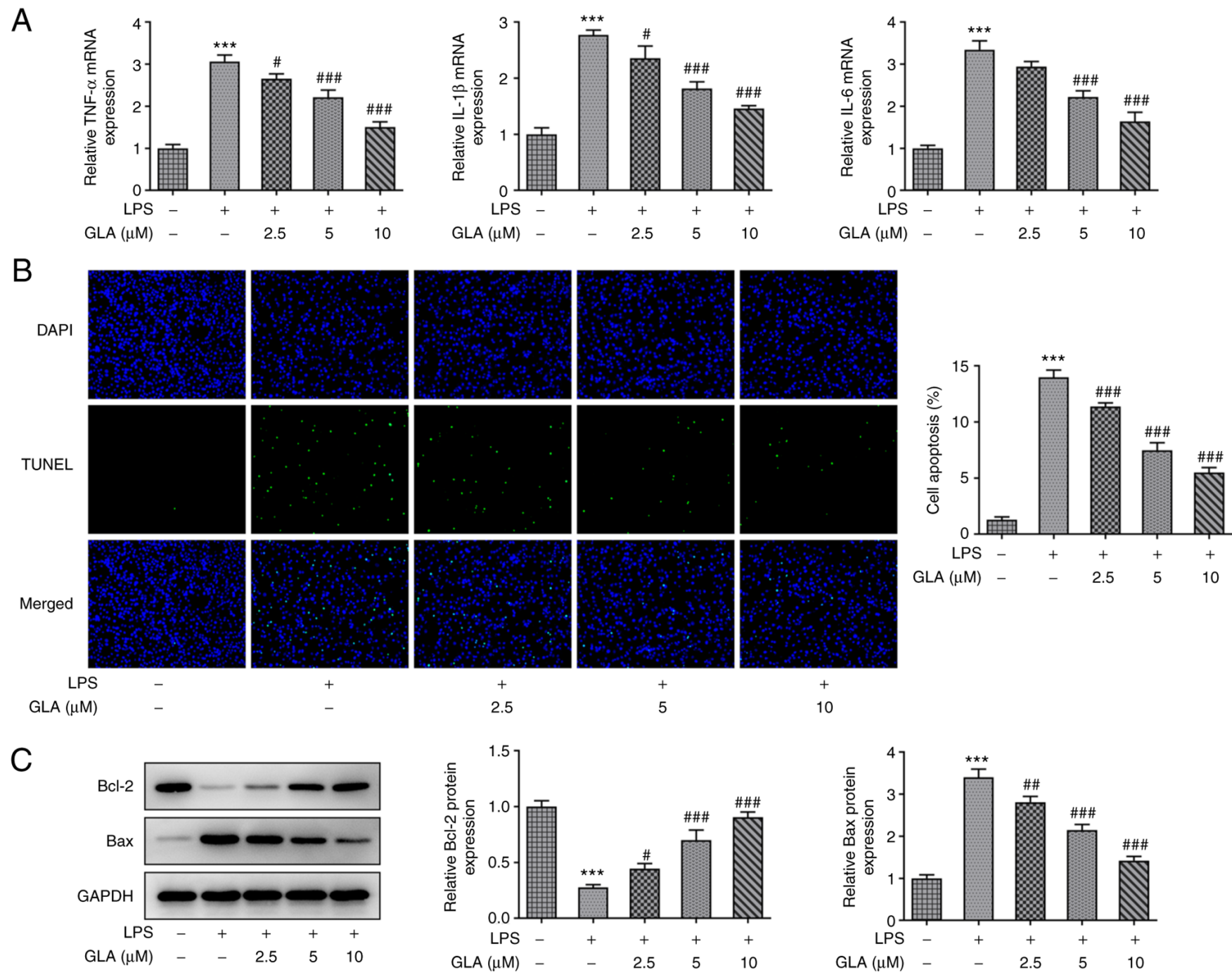

Figure 2. GLA ameliorates inflammation and apoptosis in LPS-induced hPMVECs. (A) mRNA expression level of inflammatory cytokines, (B) rate of apoptosis (magnification, $\mathrm{x} 200$ ) and (C) protein expression of apoptosis-related proteins in LPS-induced hPMVECs treated with GLA was analyzed using reverse transcription-quantitative PCR, TUNEL and western blot analysis, respectively. ${ }^{* * *} \mathrm{P}<0.001$ vs. LPS $(-)+$ GLA $(\mu \mathrm{M})(-) ;{ }^{\#} \mathrm{P}<0.05,{ }^{\# \#} \mathrm{P}<0.01$ and ${ }^{\# \# \#} \mathrm{P}<0.001$ vs. LPS (+) + GLA $(\mu \mathrm{M})(-)$. hPMVECs, human pulmonary microvascular endothelial cells; GLA, glaucocalyxin A; LPS, lipopolysaccharide.

medium and high doses of GLA did not impose cytotoxic effects on hPMVECs. Thus, 2.5, 5 and $10 \mu \mathrm{M}$ GLA concentrations were used for subsequent experiments. In addition, following LPS induction, the viability of the hPMVECs was reduced to 

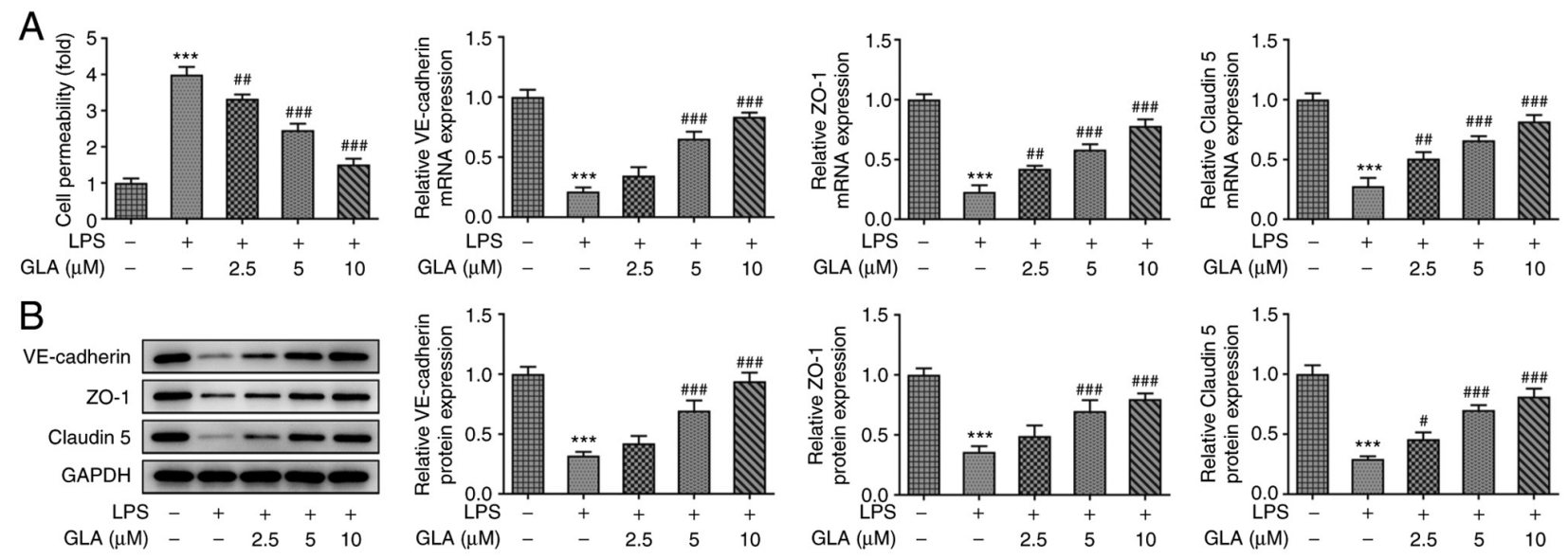

Figure 3. GLA ameliorates damage of cell permeability in LPS-induced hPMVECs. (A) Permeability of LPS-induced hPMVECs treated with GLA was detected using FITC-dextran. (B) Protein expression level of VE-cadherin, ZO-1 and claudin 5 in LPS-induced hPMVECs treated with GLA was analyzed using western blot analysis. ${ }^{* * *} \mathrm{P}<0.001$ vs. LPS (-) + GLA $(\mu \mathrm{M})(-) ;{ }^{*} \mathrm{P}<0.05,{ }^{\# \#} \mathrm{P}<0.01,{ }^{\# \# \#} \mathrm{P}<0.001$ vs. LPS $(+)+$ GLA $(\mu \mathrm{M})(-)$. hPMVECs, human pulmonary microvascular endothelial cells; GLA, glaucocalyxin A; LPS, lipopolysaccharide; VE-cadherin, vascular endothelial cadherin; ZO-1, zonula occludens-1.

A

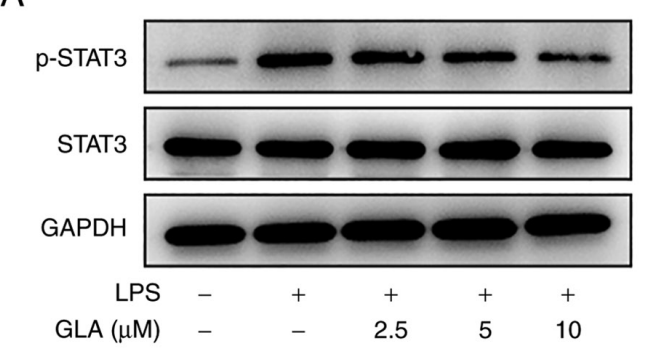

B
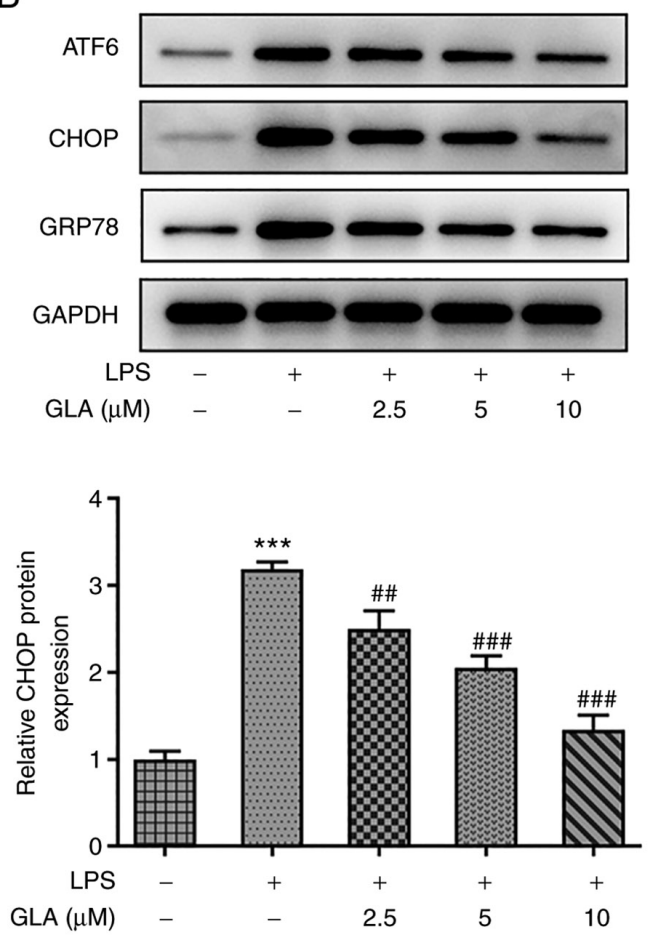
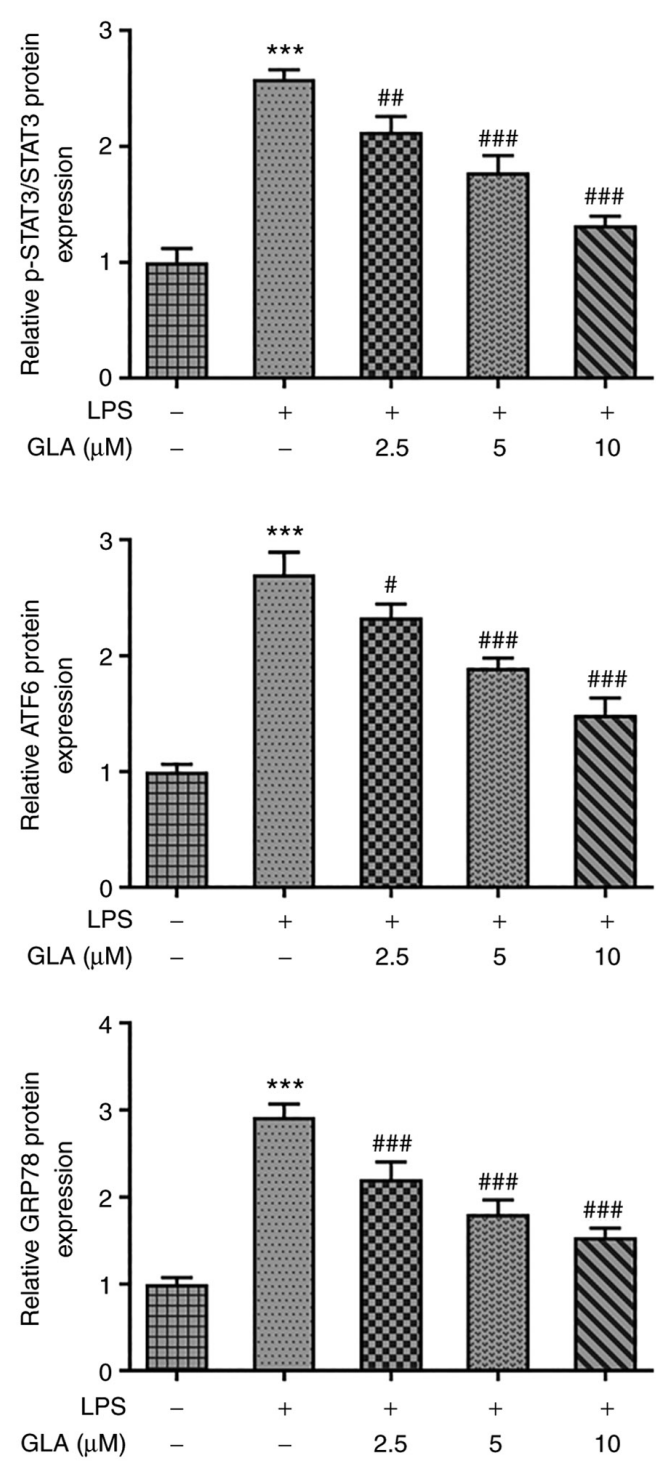

Figure 4. GLA reduces the STAT3-mediated expression of ERS-related proteins in LPS-induced hPMVECs treated with GLA. Protein expression level of (A) p-STAT3 and STAT3 and (B) ERS-related proteins in LPS-induced hPMVECs treated with GLA was analyzed using western blot analysis. ${ }^{* * *}$ P $<0.001$ vs. LPS (-) + GLA $(\mu \mathrm{M})(-) ;{ }^{\#} \mathrm{P}<0.05,{ }^{\#} \mathrm{P}<0.01,{ }^{\# \#} \mathrm{P}<0.00$ vs. LPS $(+)+$ GLA $(\mu \mathrm{M})(-)$. hPMVECs, human pulmonary microvascular endothelial cells; GLA, glaucocalyxin A; LPS, lipopolysaccharide; p, phosphorylated, ERS, endoplasmic reticulum stress; GRP78, glucose regulated protein 78; ATF6, activating transcription factor 6; CHOP, C/EBP-homologous protein; STAT3, signal transducer and activator of transcription 3. 

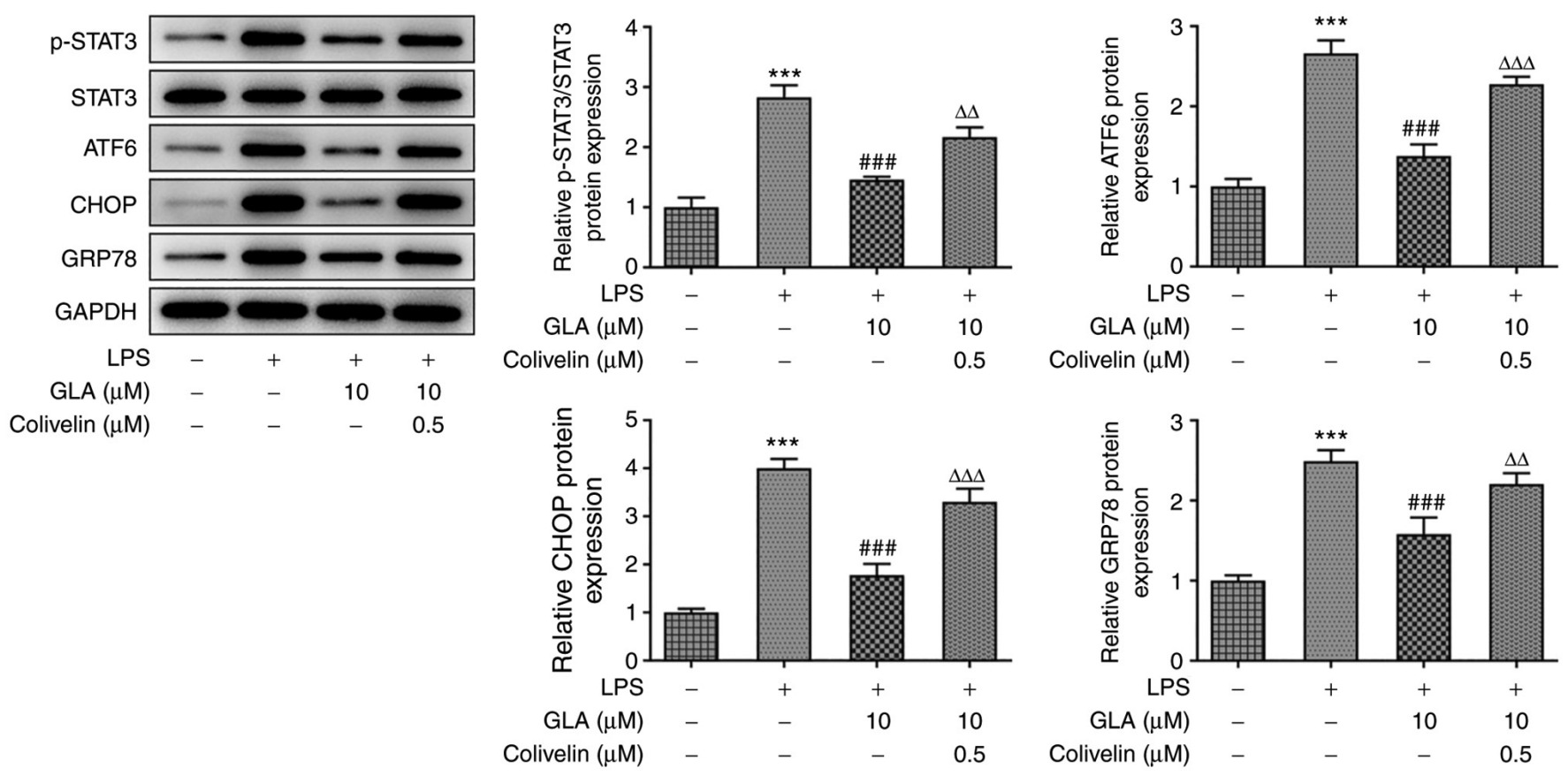

Figure 5. GLA reduces the release of ERS in LPS-induced hPMVECs by inhibiting STAT3 signal. The protein expression level of p-STAT3, STAT3 and ERS-related protein expression in LPS-induced hPMVECs co-treated with GLA and colivelin was analyzed using western blot analysis and subsequently quantified using densitometry. ${ }^{* * *} \mathrm{P}<0.001$ vs. LPS (-) + GLA $(\mu \mathrm{M})(-) ;{ }^{\# \# \#} \mathrm{P}<0.001$ vs. LPS $(+)+$ GLA $(\mu \mathrm{M})(-)+$ Colivelin $(\mu \mathrm{M})(-) ;{ }^{\Delta \Delta} \mathrm{P}<0.01,{ }^{\Delta \Delta \Delta} \mathrm{P}<0.001$ vs. LPS (+) + GLA $(\mu \mathrm{M})(10)+$ Colivelin $(\mu \mathrm{M})(-)$. hPMVECs, human pulmonary microvascular endothelial cells; GLA, glaucocalyxin A; LPS, lipopolysaccharide; p, phosphorylated, ERS, endoplasmic reticulum stress; ATF6, activating transcription factor 6; CHOP, C/EBP-homologous protein; STAT3, signal transducer and activator of transcription 3; GRP78, glucose regulated protein 78.

almost half of that in the control group, whereas this trend was reversed by increasing the dose of GLA (Fig. 1B).

GLA ameliorates inflammation, apoptosis and permeability injury in LPS-induced hPMVECs. The effect of GLA on inflammation and apoptosis in LPS-induced hPMVECs was determined. The results of RT-qPCR analysis revealed that LPS increased the mRNA expression levels of the inflammatory factors, IL- 6 , IL- $1 \alpha$ and TNF- $\alpha$, while various doses of GLA caused a reduction in these expression levels in a dose-dependent manner (Fig. 2A). The apoptosis of hPMVECs was significantly increased following treatment with LPS, and the level of apoptosis was decreased following GLA treatment (Fig. 2B). Apoptosis-related Bcl-2 protein was markedly decreased compared with control, while Bax levels were increased after LPS stimulation. These effects were considerably reversed by GLA treatment (Fig. 2C).

FITC-dextran analysis demonstrated that LPS increased the permeability of hPMVECs and reduced the mRNA and protein expression levels of VE-cadherin, ZO-1 and claudin 5. These effects were reversed following treatment with GLA (Fig. 3A and B).

Collectively, the present results demonstrated that GLA ameliorated inflammation, apoptosis and dysfunctional permeability of LPS-induced hPMVECs.

$G L A$ reduces the release of ERS-related proteins, the inflammation and the apoptosis of LPS-induced hPMVECs by inhibiting STAT3 signaling. To examine whether the function of STAT3 was associated with the underlying mechanisms by which GLA exerted protective effects on LPS-induced
hPMVECs, western blot analysis was conducted. Notably, treatment with LPS increased the protein expression levels of p-STAT3 and ERS-related proteins (ATF6, CHOP and GRP78), which were decreased following GLA treatment (Fig. 4A and B).

However, following treatment with $0.5 \mu \mathrm{M}$ colivelin in LPS-induced hPMVECs treated with GLA, the protein expression levels of p-STAT3, ATF6, CHOP and GRP78 were increased, whereas no significant changes were observed in the expression levels of total STAT3 (Fig. 5). The impacts of colivelin in STAT2 are similar to those reported in a previous study (28).

Following colivelin treatment, the mRNA expression level of inflammatory cytokines (TNF- $\alpha$, IL-1 $\beta$ and IL-6) were analyzed by RT-qPCR, while apoptosis was detected by performing a TUNEL assay. The results revealed that the mRNA expression of inflammatory cytokines and apoptosis were increased, indicating that colivelin treatment reversed the suppressive effects of GLA on inflammation and cell apoptosis (Fig. 6A and B). We also found that the activator of STAT3 significantly blunted the impacts of GLA in the expression of Bcl-2 and Bax in LPS-treated hPMVECs (Fig. 6C).

Collectively, the present results demonstrated that GLA reduced the release of ERS-related proteins in LPS-induced hPMVECs by inhibiting STAT3 signaling.

GLA ameliorates the permeability injury of LPS-induced hPMVECs by inhibiting STAT3 signaling. In order to further investigate the functions of GLA, FITC-dextran analysis was used to detect the effect of colivelin on the permeability of LPS-induced hPMVECs treated 

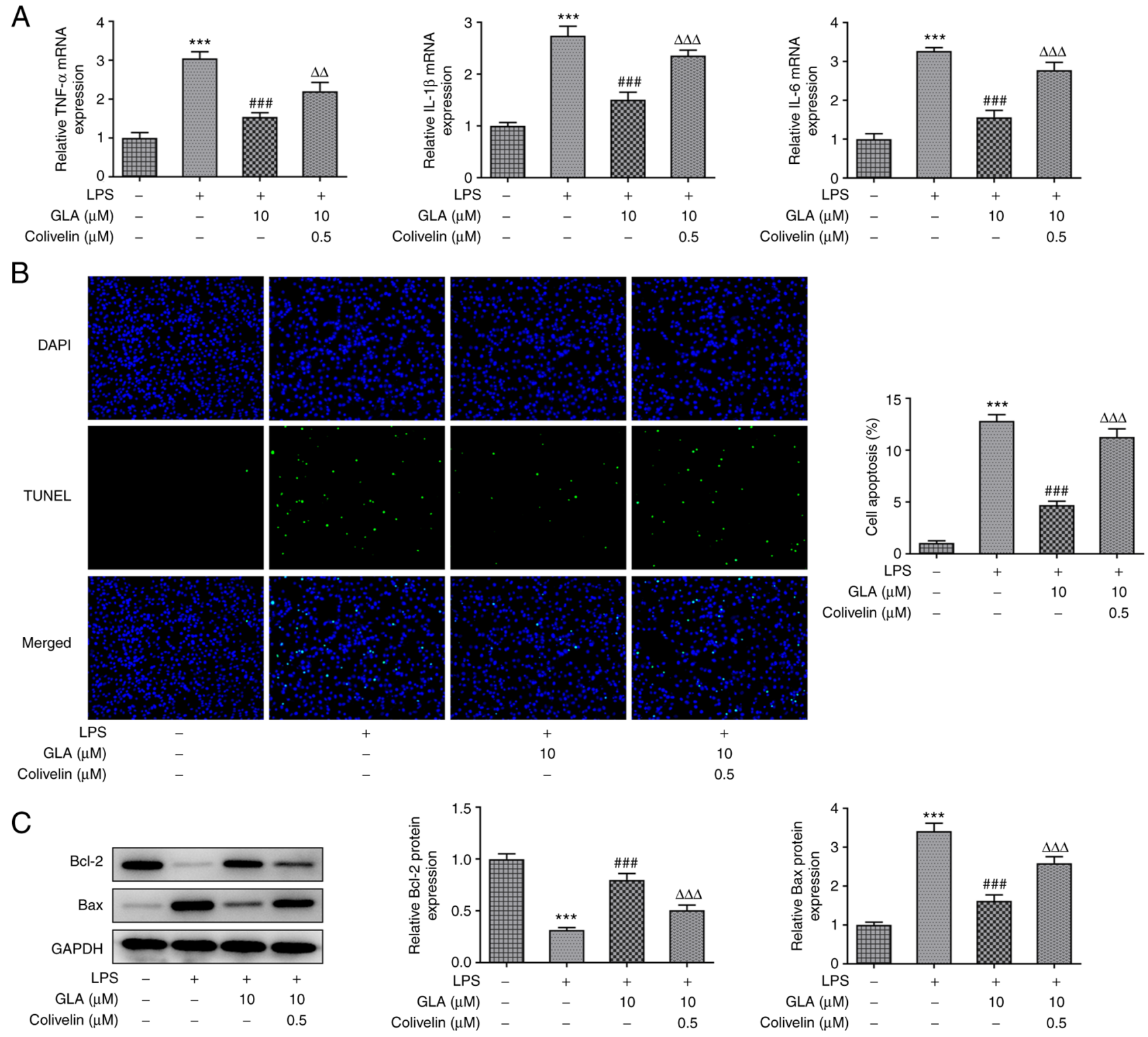

Figure 6. GLA reduces the inflammation and apoptosis in LPS-induced hPMVECs by inhibiting STAT3 signal. (A) mRNA expression level of inflammatory cytokines, (B) rate of apoptosis (magnification, x200) and (C) protein expression level of apoptosis-related proteins in LPS-induced hPMVECs co-treated with GLA and colivelin was analyzed using reverse transcription-quantitative PCR, TUNEL and western blot analysis, respectively. hPMVECs, human pulmonary microvascular endothelial cells; GLA, glaucocalyxin A; LPS, lipopolysaccharide; STAT3, signal transducer and activator of transcription 3. ${ }^{* * * *} \mathrm{P}<0.001$ vs. LPS $(-)+$ GLA $(\mu \mathrm{M})(-) ;{ }^{\# \# \#} \mathrm{P}<0.001$ vs. LPS $(+)+$ GLA $(\mu \mathrm{M})(-)+$ Colivelin $(\mu \mathrm{M})(-) .{ }^{\Delta \Lambda} \mathrm{P}<0.01$ and ${ }^{\Delta \Delta \Delta} \mathrm{P}<0.001$ vs. LPS $(+)+\mathrm{GLA}(\mu \mathrm{M})(10)+$ Colivelin $(\mu \mathrm{M})(-)$.

with GLA. The results demonstrated that the increased level of cell permeability in LPS-induced hPMVECs was reduced following treatment with GLA, whereas treatment with colivelin increased cell permeability (Fig. 7A). Furthermore, the mRNA expression levels of VE-cadherin, ZO-1 and claudin 5 were decreased following treatment with LPS, while the expression levels of these proteins were increased following GLA treatment (Fig. 7B). The observed increase in the mRNA expression levels was partially reversed following treatment with colivelin. Similar results were found in the protein expression levels of VE-cadherin, ZO-1 and claudin 5 (Fig. 7C). Collectively, the present results indicated that GLA ameliorated the permeability injury of LPS-induced hPMVECs by inhibiting STAT3 signaling.

\section{Discussion}

Pneumonia is one of the most infectious diseases affecting both children and adults, with high prevalence and morbidity rates (8). A number of studies have described the pivotal role of inflammatory cytokines and inflammation in the progression of pneumonia (29,30). Furthermore, the aggregation of inflammatory cytokines in pneumonia development induces cell apoptosis and microvascular dysfunction (31). The results of the present study initially demonstrated that GLA did not exert cytotoxicity in hPMVECs. Furthermore, hPMVECs were treated with LPS to simulate inflammation observed in the development of pneumonia. An increase in the mRNA expression levels of TNF- $\alpha$, IL-1 $\beta \alpha$ and IL-6, and an increase apoptosis were observed in hPMVECs following treatment 
A

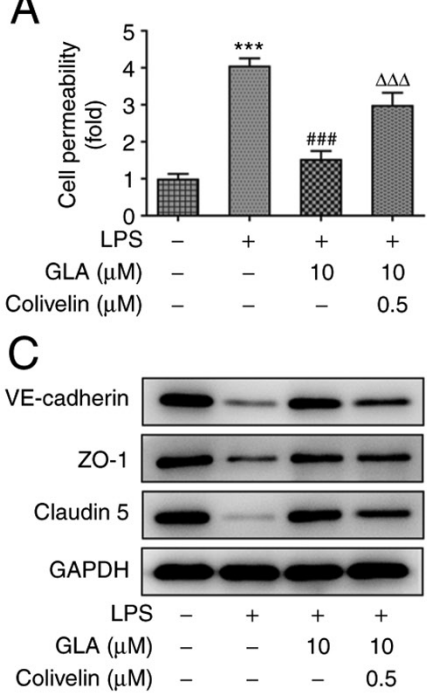

$\mathrm{B}$
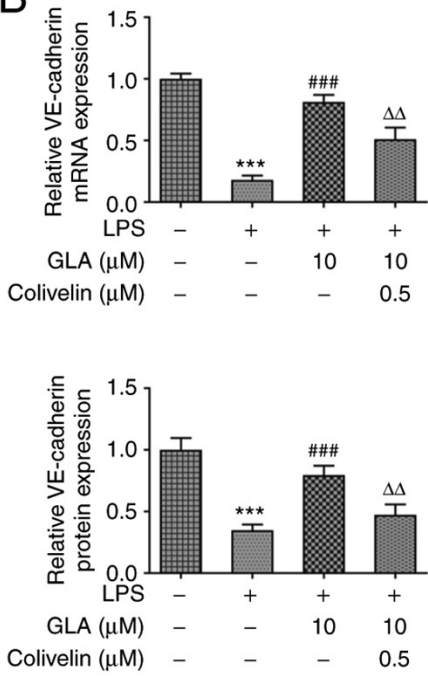
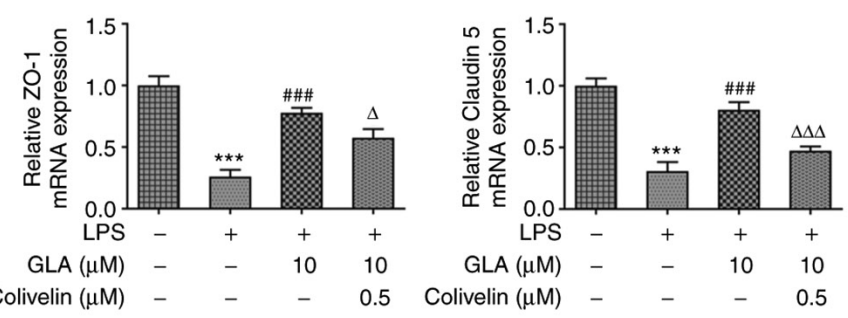

Figure 7. GLA ameliorates the damaged permeability of LPS-induced hPMVECs by inhibiting STAT3 signal. (A) Permeability of LPS-induced hPMVECs co-treated with GLA and colivelin were detected using FITC-dextran. (B) mRNA levels of VE-cadherin, ZO-1 and claudin 5 in LPS-induced hPMVECs co-treated with GLA and colivelin were analyzed using reverse transcription-quantitative PCR analysis. (C) Protein expression level of VE-cadherin, ZO-1 and claudin 5 in LPS-induced hPMVECs co-treated with GLA and colivelin was analyzed using western blot analysis. ${ }^{* * *} \mathrm{P}<0.001$ vs. LPS (-) + GLA ( $\left.\mu \mathrm{M}\right)(-)$; ${ }^{\# \# \# ~} \mathrm{P}<0.001$ vs. LPS $(+)+$ GLA $(\mu \mathrm{M})(-)+$ Colivelin $(\mu \mathrm{M})(-) ;{ }^{\Delta} \mathrm{P}<0.05,{ }^{\Delta \Delta} \mathrm{P}<0.01$ and ${ }^{\Delta \Delta \Delta} \mathrm{P}<0.001$ vs. LPS $(+)+$ GLA $(\mu \mathrm{M})(10)+$ Colivelin $(\mu \mathrm{M})(-)$. hPMVECs, human pulmonary microvascular endothelial cells; GLA, glaucocalyxin A; LPS, lipopolysaccharide; VE-cadherin, vascular endothelial cadherin; ZO-1, zonula occludens-1.

with LPS, which is consistent with previous studies $(32,33)$. Whereas treatment with GLA resulted in decreased inflammation and apoptosis in LPS-induced hPMVECs, as indicated by the reduced mRNA expression levels of inflammatory cytokines and a decrease in the rate of apoptosis. Loss of endothelial barrier integrity is another key characteristic of pneumonia (34), and the results from the present study demonstrated that the enhanced endothelial permeability stimulated by LPS was reversed following treatment with GLA.

STAT3 is a transcription factor that exerts biological effects in immunity and inflammation (35). The results of a previous study revealed the participation of STAT3 in bacterial Escherichia coli pneumonia, as the protein levels of p-STAT3 were markedly decreased in IL-6-deficient mice, highlighting STAT3 as a potential therapeutic target for the treatment of this disease (36). Furthermore, a reduction in the protein levels of STAT3 could aggregate lung injury during pneumonia (37). These findings associated STAT3 signaling with the severity and progression of pneumonia. Another previous study also revealed that the amelioration of ERS via caffeine treatment mitigated hyperoxia-induced lung injury (38). In the present study, STAT3 protein expression level was markedly elevated in response to LPS treatment for $24 \mathrm{~h}$ in hPMVECs, which is consistent with the findings of a previous study (20). Furthermore, GLA treatment suppressed the phosphorylation of STAT3 and reduced the protein expression levels of ERS-associated proteins in the present study, which further suggests that GLA may alleviate ERS by regulating STAT3 signaling in pneumonia. To verify this hypothesis, hPMVECs were treated with colivelin to determine the changes in the protein expression levels of p-STAT3 and ERS-associated proteins. The suppressive effects of GLA on both the protein expression levels of p-STAT3 and ERS-associated proteins were reversed following treatment with colivelin. STAT3 was also suggested to be essential for the inflammation and pathogenesis of acute lung injury by regulating the expression of inflammation- and immune-related proteins (39). The present study indicated that the GLA-induced reduction in the mRNA expression level of inflammatory cytokines was reversed following treatment with colivelin in LPS-induced hPMVECs, indicating that STAT3 may indirectly modulate the inflammatory response by mediating associated cytokines to affect the progression of pneumonia. In addition, further investigation also confirmed that the GLA-induced levels of apoptosis and permeability injury in LPS-induced hPMVECs were reversed following treatment with colivelin, demonstrating that GLA inhibited apoptosis and permeability injury in LPS-induced hPMVECs by inhibiting STAT3 signaling. It was reported that the mTOR/STAT3 pathway or the JAK2/STAT3 signaling pathway was associated with lung injury, which provides an insight on understanding how GLA regulates STAT3 $(40,41)$, which still requires further investigation.

In conclusion, GLA alleviated LPS-induced inflammation, apoptosis and permeability injury of hPMVECs by inhibiting STAT3 signaling, which revealed the therapeutic value of GLA in the treatment of pneumonia. The functional effects of GLA in LPS-induced hPMVECs were explored in vitro, and further studies should aim to expand these investigations into in vivo models.

\section{Acknowledgements}

No applicable.

\section{Funding}

The present study was supported by Zhongshan Medical Scientific Research Item (grant no. 2018A020169). 


\section{Availability of data and materials}

The datasets used and/or analyzed during the current study are available from the corresponding author on reasonable request.

\section{Authors' contributions}

JWC and KJZ made substantial contributions to the conception and design of the current study. JWC, MLL, SFF and YYL performed the experiments and collected data. JWC and KJZ interpreted the data, and drafted and revised the manuscript for important intellectual content. JWC and KJZ confirm the authenticity of the raw data. All authors read and approved the final manuscript.

\section{Ethics approval and consent to participate}

Not applicable.

\section{Patient consent for publication}

Not applicable.

\section{Competing interests}

The authors declare that they have no competing interests.

\section{References}

1. Li W, An X, Fu M and Li C: Emergency treatment and nursing of children with severe pneumonia complicated by heart failure and respiratory failure: 10 Case reports. Exp Ther Med 12: 2145-2149, 2016.

2. Korppi M: Diagnosis and treatment of community-acquired pneumonia in children. Acta Paediatr 101: 702-704, 2012.

3. Simonetti AF, Viasus D, Garcia-Vidal C and Carratalà J: Management of community-acquired pneumonia in older adults. Ther Adv Infect Dis 2: 3-16, 2014.

4. Mattila JT, Fine MJ, Limper AH, Murray PR, Chen BB and Lin PL: Pneumonia. Treatment and diagnosis. Ann Am Thorac Soc 11 (Suppl 4): S189-S192, 2014.

5. Dreyfuss D and Ricard JD: Acute lung injury and bacterial infection. Clin Chest Med 26: 105-112, 2005.

6. Zhang J, Mao F, Zhao G, Wang H, Yan X and Zhang Q: Long non-coding RNA SNHG16 promotes lipopolysaccharides-induced acute pneumonia in A549 cells via targeting miR-370-3p/IGF2 axis. Int Immunopharmacol 78: 106065, 2020.

7. Zeng M, Sang W, Chen S, Chen R, Zhang H, Xue F, Li Z, Liu Y, Gong Y, Zhang $\mathrm{H}$ and Kong X: 4-PBA inhibits LPS-induced inflammation through regulating ER stress and autophagy in acute lung injury models. Toxicol Lett 271: 26-37, 2017.

8. Zhang Y, Zhu Y, Gao G and Zhou Z: Knockdown XIST alleviates LPS-induced WI-38 cell apoptosis and inflammation injury via targeting miR-370-3p/TLR4 in acute pneumonia. Cell Biochem Funct 37: 348-358, 2019.

9. Zhou Z, Zhu Y, Gao G and Zhang Y: Long noncoding RNA SNHG16 targets miR-146a-5p/CCL5 to regulate LPS-induced WI-38 cell apoptosis and inflammation in acute pneumonia. Life Sci 228: 189-197, 2019.

10. Zang L, Song Y, Yu F and Liu X: Emodin relieved lipopolysaccharide-evoked inflammatory damage in WI-38 cells by up-regulating taurine up-regulated gene 1. Biofactors 46: $860-868,2020$.

11. Dong Z, Gao Q and Guo H: Glaucocalyxin A attenuates the activation of hepatic stellate cells through the TGF- $\beta 1 / \mathrm{smad}$ signaling pathway. DNA Cell Biol 37: 227-232, 2018.

12. Xiang Z, Wu X, Liu X and Jin Y: Glaucocalyxin A: A review. Nat Prod Res 28: 2221-2236, 2014.
13. Hou X, Xu G, Wang Z, Zhan X, Li H, Li R, Shi W, Wang C, Chen Y, Ai Y, et al: Glaucocalyxin A alleviates LPS-mediated septic shock and inflammation via inhibiting NLRP3 inflammasome activation. Int Immunopharmacol 81: 106271, 2020.

14. Liu X, Xu D, Wang Y, Chen T, Wang Q, Zhang J, You T and Zhu L: Glaucocalyxin A ameliorates myocardial ischemia-reperfusion injury in mice by suppression of microvascular thrombosis. Med Sci Monit 22: 3595-3604, 2016.

15. Wang M, Zhang T, Li L, Xie Q, Wang Y, Li Y and Chen Z: Protective effects of HY1702 on lipopolysaccharide-induced mild acute respiratory distress syndrome in mice. Eur J Pharmacol 887: 173563, 2020.

16. Yang F, Cao Y, Zhang J, You T and Zhu L: Glaucocalyxin A improves survival in bleomycin-induced pulmonary fibrosis in mice. Biochem Biophys Res Commun 482: 147-153, 2017.

17. Peng Z, Zhang R, Pan L, Pei H, Niu Z, Wang H, Lv J and Dang X: Glaucocalyxin A PRotects H9c2 cells against hypoxia/reoxygenation-induced injury through the activation of Akt/ $\mathrm{Nrf} 2 / \mathrm{HO}-1$ pathway. Cell Transplant 29: 963689720967672, 2020.

18. Zhu S, Zhang J and Lv Y: Glaucocalyxin A inhibits hydrogen peroxide-induced oxidative stress and inflammatory response in coronary artery smooth muscle cells. Clin Exp Pharmacol Physiol 47: 765-770, 2020.

19. Mao M, Zhang T, Wang Z, Wang H, Xu J, Yin F, Wang G, Sun M, Wang Z, Hua Y and Cai Z: Glaucocalyxin A-induced oxidative stress inhibits the activation of STAT3 signaling pathway and suppresses osteosarcoma progression in vitro and in vivo. Biochim Biophys Acta Mol Basis Dis 1865: 1214-1225, 2019.

20. Zhao J, Yu H, Liu Y, Gibson SA, Yan Z, Xu X, Gaggar A, Li PK, Li C, Wei S, et al: Protective effect of suppressing STAT3 activity in LPS-induced acute lung injury. Am J Physiol Lung Cell Mol Physiol 311: L868-L880, 2016.

21. Wang L, Astone M, Alam SK, Zhu Z, Pei W, Frank DA, Burgess SM and Hoeppner LH: Suppressing STAT3 activity protects the endothelial barrier from VEGF-mediated vascular permeability. bioRxiv: doi: 10.1101/2020.10.27.358374.

22. Meng J, Liu K, Shao Y, Feng X, Ji Z, Chang B, Wang Y, Xu L and Yang G: ID1 confers cancer cell chemoresistance through STAT3/ATF6-mediated induction of autophagy. Cell Death Dis 11: 137, 2020.

23. Jiang CQ, Ma LL, Lv ZD, Feng F, Chen Z and Liu ZD: Polydatin induces apoptosis and autophagy via STAT3 signaling in human osteosarcoma MG-63 cells. J Nat Med 74: 533-544, 2020.

24. Qs L, K C, Ap L, F X, Qw H, Z L, Qh Y, YI W, Zz Z and J Z: Roles of $\mathrm{M}_{3}$ receptor in the effect of penehyclidine hydrochloride upregulated beta-arrestin-1 expression in LPS-stimulated HPMVEC. J Recept Signal 39: 39-44, 2019.

25. Livak KJ and Schmittgen TD: Analysis of relative gene expression data using real-time quantitative PCR and the 2(-Delta Delta C(T)) method. Methods 25: 402-408, 2001.

26. Vallverdú J, Martínez García de la Torre RA, Mannaerts I, Verhulst S, Smout A, Coll M, Ariño S, Rubio-Tomás T, Aguilar-Bravo B, Martínez-Sánchez C, et al: Directed differentiation of human induced pluripotent stem cells to hepatic stellate cells. Nat Protoc 16: 2542-2563, 2021.

27. Zhou Y, Li P, Goodwin AJ, Cook JA, Halushka PV, Chang E, Zingarelli B and Fan $\mathrm{H}$ : Exosomes from endothelial progenitor cells improve outcomes of the lipopolysaccharide-induced acute lung injury. Crit Care 23: 44, 2019.

28. Fang YY and Zhang JH: MFG-E8 alleviates oxygen-glucose deprivation-induced neuronal cell apoptosis by STAT3 regulating the selective polarization of microglia. Int J Neurosci 131: 15-24, 2021.

29. Mizgerd JP: Inflammation and pneumonia: Why are some more susceptible than others? Clin Chest Med 39: 669-676, 2018.

30. Jae SY, Heffernan KS, Kurl S, Kunutsor SK, Kim CH, Johnson BD, Franklin BA and Laukkanen JA: Cardiorespiratory fitness, inflammation, and the incident risk of pneumonia. J Cardiopulm Rehabil Prev 41: 199-201, 2021.

31. Redente EF, Keith RC, Janssen W, Henson PM, Ortiz LA, Downey GP, Bratton DL and Riches DW: Tumor necrosis factor- $\alpha$ accelerates the resolution of established pulmonary fibrosis in mice by targeting profibrotic lung macrophages. Am J Respir Cell Mol Biol 50: 825-837, 2014.

32. Jiang Z, Shen J, Ding J, Yuan Y, Gao L, Yang Z and Zhao X: USP18 mitigates lipopolysaccharide-induced oxidative stress and inflammation in human pulmonary microvascular endothelial cells through the TLR4/NF- $\mathrm{B} / \mathrm{ROS}$ signaling. Toxicol In Vitro 75: 105181, 2021. 
33. Zhang D, Zhou J, Ye LC, Li J, Wu Z, Li Y and Li C: Autophagy maintains the integrity of endothelial barrier in LPS-induced lung injury. J Cell Physiol 233: 688-698, 2018.

34. Wang T, Yegambaram M, Gross C, Sun X, Lu Q, Wang H, Wu X, Kangath A, Tang H, Aggarwal S and Black SM: RAC1 nitration at $\mathrm{Y}^{32}$ IS involved in the endothelial barrier disruption associated with lipopolysaccharide-mediated acute lung injury. Redox Biol 38: 101794, 2021.

35. Lee H, Jeong AJ and Ye SK: Highlighted STAT3 as a potential drug target for cancer therapy. BMB Rep 52: 415-423, 2019.

36. Jones MR, Quinton LJ, Simms BT, Lupa MM, Kogan MS and Mizgerd JP: Roles of interleukin-6 in activation of STAT proteins and recruitment of neutrophils during Escherichia coli pneumonia. J Infect Dis 193: 360-369, 2006.

37. Quinton LJ, Jones MR, Robson BE, Simms BT, Whitsett JA and Mizgerd JP: Alveolar epithelial STAT3, IL-6 family cytokines, and host defense during Escherichia coli pneumonia. Am J Respir Cell Mol Biol 38: 699-706, 2008.

38. Teng RJ, Jing X, Michalkiewicz T, Afolayan AJ, Wu TJ and Konduri GG: Attenuation of endoplasmic reticulum stress by caffeine ameliorates hyperoxia-induced lung injury. Am J Physiol Lung Cell Mol Physiol 312: L586-L598, 2017.
39. Severgnini M, Takahashi S, Rozo LM, Homer RJ, Kuhn C, Jhung JW, Perides G, Steer M, Hassoun PM, Fanburg BL, et al: Activation of the STAT pathway in acute lung injury. Am J Physiol Lung Cell Mol Physiol 286: L1282-L1292, 2004.

40. Meng SS, Guo FM, Zhang XW, Chang W, Peng F, Qiu HB and Yang Y: mTOR/STAT-3 pathway mediates mesenchymal stem cell-secreted hepatocyte growth factor protective effects against lipopolysaccharide-induced vascular endothelial barrier dysfunction and apoptosis. J Cell Biochem 120: 3637-3650, 2019.

41. Zhang L, Lu P, Guo X, Liu T, Luo X and Zhu YT: Inhibition of JAK2/STAT3 signaling pathway protects mice from the DDP-induced acute kidney injury in lung cancer. Inflamm Res 68: 751-760, 2019.

This work is licensed under a Creative Commons Attribution-NonCommercial-NoDerivatives 4.0 International (CC BY-NC-ND 4.0) License. 